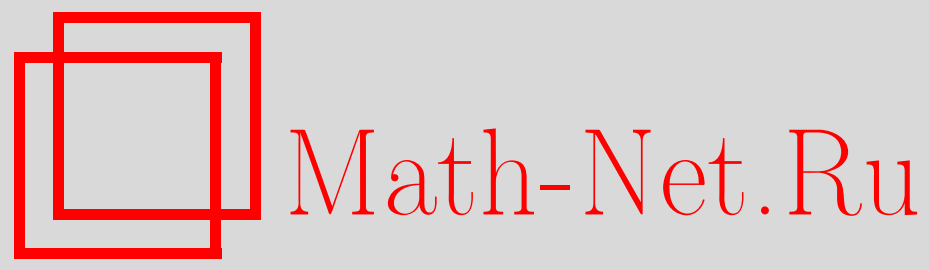

А. М. Вершик, Статистическая механика комбинаторных разбиений и их предельные конфигурации, Функи. анализ и его прил., 1996, том 30, выпуск 2, 19-39

DOI: https://doi.org/10.4213/faa519

Использование Общероссийского математического портала MathNet.Ru подразумевает, что вы прочитали и согласны с пользовательским соглашением http://www . mathnet.ru/rus/agreement

Параметры загрузки:

IP : 18.234 .197 .8

26 апреля 2023 г., 08:31:58

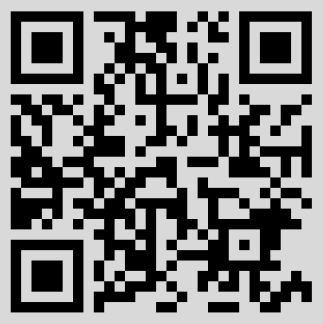


Функииональньй анализ и его приложения

1996, т. 30, вып. 2, с. 19-39

УДК $514.172 .45,519.2$

\title{
Статистическая механика комбинаторных разбиений и их предельные конфигурации
}

\author{
(c) 1996. А. М. Вершик ${ }^{1}$
}

Я. Г. Синаю кего 60-летию

\section{§. Введение}

Под разбиением в этой статье мы имеем в виду представление натурального числа или вектора с натуральными координатами в виде суммы таких же неупорядоченных слагаемых. Этот объект со времен Эйлера занимает специалистов по разным областям математики - теории чисел, комбинаторике, теории вероятностей и др. Новая задача, относящаяся к асимптотической теории разбиений, появившаяся некоторое время тому назад, - задача об асимптотической (предельной) конфигурации или форме разбиений относительно той или иной статистики. Вот ее простейшая постановка (см. $\S 1$ ). Пусть на множестве $\mathcal{P}_{n}$ всех разбиений числа $n$ задана равномерная мера $\mu^{n}: \mu^{n}(\lambda)=p(n), \lambda \in \mathcal{P}_{n}$, где $p(\cdot)$ — функция Эйлера (partitio numerorum); нельзя ли нормировать разбиения таким образом, чтобы в надлежащем пространстве меры $\mu^{n}$ слабо сходились к некоторой предельной мере на обобщенных разбиениях? Будет ли этот предел вырожденным ( $\delta$-мерой)? В последнем случае он сосредоточен на предельной конфигурации. Положительный ответ, а также явные формулы предельных конфигураций для ряда статистик даны ниже. В этой работе мы продолжаем изучение подобных задач (см. обзор [1]) и подчеркиваем тесную связь со статистической физикой идеального газа. Проблема нахождения предельной конфигурации в таком контексте чрезвычайно органична для традиционных статистик (Бозе, Ферми и др.), хотя, насколько мне известно, ранее не изучалась. Она может быть решена с помощью вариации классических средств (см. далее) и интерпретируется как задача о предельном распределении общей энергии системы по энергетическому спектру. Однако наш предельный переход буквально не адекватен термодинамическому предельному переходу (N-V-пределу): фактически мы сводим задачу о предельном распределении энергии к случаю фиксированного объема, когда энергия стремится к бесконечности, а рост ее не обязательно согласован с ростом числа частиц. Если число слагаемых (частиц) не фиксировано (случай, аналогичный статистике фотонов), то естественный рост числа частиц уже автоматически определяется статистикой. Классический $\mathrm{N}-\mathrm{V}$-предел можно уложить в нашу схему, выбирая рост числа слагаемых специальным образом

\footnotetext{
${ }^{1}$ Работа поддержана РФФИ (грант 94-01-00921) и Международным научным фондом (грант MVQ000).
} 
(см. далее), но соответствующие комбинаторные задачи пока мало изучены; они связаны также с поведением сверток распределений на полугруппе разбиений. См. замечание в конце $\S 1$.

С математической точки зрения мы изучаем слабую сходимость мер в надлежащем пространстве предельных распределений (компакт $\mathcal{D})$, и основное утверждение гласит, что в ряде случаев пределы мер специального вида (мультипликативные меры, например, равномерные меры на разбиениях) на множестве разбиений фиксированного числа $n$ (=энергии) при $n \rightarrow \infty$ совпадают с пределами некоторой смеси этих мер по различным $n$, и, таким образом, их предельные конфигурации совпадают. Собственно, в этом и состоит идея применения большого канонического ансамбля, снабженного гиббсовской мерой: его изучение проще, чем изучение микроканонического ансамбля, так как числа заполнений становятся независимыми относительно гиббсовской меры. В комбинаторике эта идея неявно использовалась (см. [2-4]), но задача о предельной конфигурации не ставилась. Утверждение, высказанное выше, можно назвать слабой эквивалентностью большого и малого канонических ансамблей, но, учитывая замечание о различии пределов, оно имеет более общий смысл, чем эквивалентность ансамблей в статистической механике. Тем не менее методы аналогичны: и там, и в нашем случае для нахождения пределов и обоснования корректности можно использовать как метод перевала (Дарвина-Фаулера [5]), так и метод локальной предельной теоремы (первым, видимо, это заметил А. Я. Хинчин, см. [6]). Стоит лишь подчеркнуть, что основная трудность в обосновании - одна и та же, хотя преодолевается по-разному. В задачах теории чисел вероятностную методику для нахождения асимптотики числовых функций использовал Г. Фрейман [7]. Прекрасный пример того, как работает вероятностный метод в задаче о векторных разбиениях и выпуклых кривых, решенной в [8, 9], был дан в работе Я. Г. Синая [10].

Задача о предельной конфигурации разбиений была поставлена впервые автором в связи с асимптотической теорией классических групп и их представлений, в частности симметрической группы [1], но ее следует рассматривать и в более общем контексте известных задач о случайном росте конфигураций. Заметим, что комбинаторные схемы, встречающиеся в комбинаторике, теории групп и представлений, а также в аддитивной теории чисел, гораздо разнообразнее, чем обычные статфизические модели идеального газа. В частности, эргодичность большого и малого ансамблей не всегда имеет место в естественных задачах. Стимулирующий характер этих задач проявляется, в частности, в том, как взаимодействуют различные методы их решения. Например, вариационный принцип (см. $[8,16])$, состоящий в нахождении предельной конфигурации как минимума некоторого функционала, привел к появлению нового типа вариационных задач (cм. 6 ).

В этой статье мы приводим основные формулировки и серию результатов о предельной конфигурации. Подробное их изложение составит другую работу. В работе над данной статьей автор многократно вспоминал свои беседы с Я. Г. Синаем, который на протяжении многих лет пропагандировал идеи сближения статистической физики и традиционных разделов математики. Автору были полезны также дискуссии с Р. А. Минлосом и в особенности с безвременно 
скончавшимся в 1995 г. замечательным математиком Л. Р. Добрушиным, который неоднократно делился с автором своим пониманием статистической физики и смежных математических теорий. Наконец, вопросы слушателей спецкурса «Разбиения», который автор читал в СПбГУ в осеннем семестре 1995/96 гг. и в который был включен обзор по проблематике этой работы, также стимулировали обдумывание ряда вопросов.

\section{§1. Постановка вопроса, определения, терминология}

Обозначим через $\mathcal{P}_{n}=\{\lambda: \lambda \vdash n\}$ множество всех разбиений натурального числа $n \geqslant 0$ в сумму неупорядоченных натуральных ненулевых слагаемых, $\lambda=\left(\lambda_{1}, \ldots, \lambda_{N}\right)$; сумма будет обозначаться через $n(\lambda)$, а число (ненулевых) слагаемых — через \# $\lambda$ или $N(\lambda)$. Множество разбиений числа $n$ с $N$ слагаемыми обозначим через $\mathcal{P}_{n, N}, \mathcal{P}_{n}=\bigcup_{N} \mathcal{P}_{n, N} \cdot$ Наконец, $\mathcal{P}=\bigcup_{n} \mathcal{P}_{n}-$ несвязное объединение; его можно назвать большим каноническим ансамблем разбиений, $\mathcal{P}_{n, N}$ - мальм или микроканоническим, а $\mathcal{P}_{n}$ - каноническим ансамблем.

Свяжем с $\lambda \in \mathcal{P}_{n}$ набор чисел $r_{1}(\lambda), \ldots, r_{t}(\lambda)$, где $r_{k}(\lambda)=\#\left\{j: \lambda_{j}=k\right\}$ - число слагаемых в разбиении $\lambda$, равных $k$; будем называть $r_{k}(\lambda)$ числами заполнения; очевидно, что вектор $\left(r_{i}(\lambda)\right)$ полностью описывает разбиение $\lambda$ это есть распределение разбиения. Ясно, что $n(\lambda)=\sum_{k} k r_{k}(\lambda), \# \lambda=N(\lambda)=$ $\sum_{k} r_{k}(\lambda)$. Напомним, что \# $\mathcal{P}_{n}=p(n)$ - функция Эйлера с производящей функцией

$$
\prod_{k=1}^{\infty} \frac{1}{1-x^{k}}=\sum_{n=0}^{\infty} p(n) x^{n} .
$$

Сопоставим разбиению $\lambda \in \mathcal{P}_{n}$ следующую функщию $\varphi_{\lambda}$ на $[0, \infty)$ :

$$
\varphi_{\lambda}(t)=\sum_{k \geqslant t} r_{k}(\lambda), \quad 0 \leqslant t<\infty .
$$

Очевидно, что $\varphi_{\lambda}$ есть ступенчатая функция, непрерывная справа, и $\int_{0}^{\infty} \varphi_{\lambda}(t) d t$ $=n(\lambda)$. Замыкание внутренности ее подграфика называется диаграммой Юнга, отвечающей разбиению $\lambda$. Пусть $a>0$; нормированной на $a$ функцией назовем функцию

$$
\widetilde{\varphi}_{\lambda}(t)=\frac{a}{n} \sum_{k \geqslant a t} r_{k}(\lambda)=\frac{a}{n} \varphi_{\lambda}(a t),
$$

$\int_{0}^{\infty} \widetilde{\varphi}_{\lambda}(t) d t=1$; функщия $\widetilde{\varphi}$ есть образ функции $\varphi$ при преобразовании плоскости $(t, \varphi) \mapsto(a t, \varphi n / a)$. Выбор нормировки $a$ (скейлинга) будет обсуждаться далее. Обозначим отображение $\lambda \mapsto \widetilde{\varphi}_{\lambda}$ через $\tau_{a}$. Введем состоящее из троек пространство $\mathcal{D}=\left\{\left(\alpha_{0}, \alpha_{\infty}, p(\cdot)\right): \alpha_{0}, \alpha_{\infty} \in \mathbb{R}_{+}, p \in L_{+}^{1}\left(\mathbb{R}_{+}\right)\right.$- неотрицательная монотонно невозрастающая функщия на $\left.[0, \infty), \alpha_{0}+\alpha_{\infty}+\int_{0}^{\infty} p(t) d t=1\right\}$.

Можно представлять себе числа $\alpha_{0}$ и $\alpha_{\infty}$ как заряды меры в 0 и $+\infty$ соответственно, и, таким образом, элементы из $\mathcal{D}$ есть меры $\alpha_{0} \delta_{0}+\alpha_{\infty} \delta_{\infty}+p d t$ на $[0, \infty]$ с двумя возможными атомами на концах и монотонной плотностью по 
лебеговой мере на $(0, \infty)$. Меры $\delta_{0}$ и $\delta_{\infty}$ будем называть тривиальными элементами множества $\mathcal{D}$, меры, для которых $p \neq 0$, - собственными элементами, а меры с $\alpha_{0}=\alpha_{\infty}=0$ - непрерывными элементами.

Снабдим $\mathcal{D}$ топологией равномерной сходимости на компактах, лежащих в $(0, \infty)$, и обычной сходимости величин зарядов в 0 и $\infty$.

Лемма 1.1. В этой топологии $\mathcal{D}$ есть метризуемый компакт.

Доказательство компактности следует из равномерной ограниченности и равностепенной абсолютной непрерывности монотонных функций на интервалах $\left[t_{0}, t_{1}\right], t_{0}>0$.

СЛЕдСТВИЕ. Бесконечная совокупность борелевских нормированных неотрицательньх мер на $\mathcal{D}$ имеет непустое множество предельньх точек в слабой топологии мер.

Очевидно, что образ множества $\mathcal{P}_{n}$ при отображении $\tau_{a}: \mathcal{P}_{n} \rightarrow \mathcal{D}$ состоит из мер с нулевыми $\alpha_{0}$ и $\alpha_{\infty}$ и кусочно-постоянной плотностью.

Предположим, что на $\mathcal{P}_{n}$ задана вероятностная мера (статистика) $\mu^{n}$; тогда формула $\left(\tau_{a}^{*} \mu^{n}\right)(A) \equiv \mu^{n}\left(\tau_{a}^{-1} A\right), A \in \mathcal{D}$, определяет ее образ при отображении $\tau_{a}$. Мы изучаем следующий вопрос: существует ли для данной последовательности мер $\mu^{n}$ на $\mathcal{P}_{n}$ последовательность чисел $a_{n}$, при которой последовательность образов $\tau_{a}^{*} \mu^{n}$ слабо сходится $\kappa$ мере, носитель которой нетривиален (m.е. не есть заряд в 0 или $\infty)$. Если такая нормировка (скейлинг) существует и предельная мера непрерывна, то эта нормировка в существенном единственна. Точнее, если две нормировки $\left\{a_{n}\right\}$ и $\left\{a_{n}^{\prime}\right\}$ дают нетривиальные предельные меры, то, во-первых, они эквивалентны, т. е. $a_{n} / a_{n}^{\prime} \rightarrow 1$, а во-вторых, эти предельные меры равны. Если при этом предельная мера вырожденна, т. е. сосредоточена в одном (непрерывном) элементе $C \in \mathcal{D}$, то этот элемент называется предельной конфигурацией или предельной формой (limit shape) случайных разбиений со статистиками $\left\{\mu^{n}\right\}$ и нормировкой $\left\{a_{n}\right\}$, этот случай назовем эргодическим. Итак, задача состоит в том, чтобы для данной последовательности мер $\mu^{n}$ на $\mathcal{P}_{n}$ найти такую последовательность чисел $a_{n}$, что скейлинговые образы $\tau_{a_{n}}^{*} \mu^{n}$ слабо сходятся к некоторой мере $\bar{\mu}$ в $\mathcal{D}$ (или доказать несуществование такой нормировки). Положительный ответ на этот вопрос позволяет высказывать утверждения типа: «почти все разбиения асимптотически таковы, что сумма слагаемых, больших чем $t a_{n}$, составляет данную долю от всей суммы» (в эргодическом случае) или «вероятность того, что эта доля принадлежит данному интервалу, равна заданному числу» (неэргодический случай). Огромное число задач асимптотической комбинаторики сводится к этой проблеме; в общей постановке она слишком широка; мы изучаем здесь специальный класс мер $\mu^{n}$ - мультипликативные статистики на $\mathcal{P}$. В терминах статистической физики разбиение $\lambda \in \mathcal{P}_{n}$ есть набор частищ (точнее, их энергий), сумма энергий которых равна $n(=E)$, а число частиц с данной энергией $k$ есть число заполнения $r_{k}(\lambda)$; энергии частищ и всей системы есть натуральные числа, что в теории идеального газа не является серьезным ограничением, поскольку они являются собственными числами оператора Лапласа в кубе или на торе. Общее число частищ равно $\sum r_{k}(\lambda)=N(\lambda)$. В случае идеального газа система полностью определена, если задан набор импульсов всех частиц, т. е. числа заполнения. Множество $\mathcal{P}_{n, N}$ интерпретируется как множество конфигураций 
$\lambda$ с заданными суммарной энергией и числом частиц, т.е. микроканонический ансамбль (в единичном объеме). Однако множество частиц с данной энергией в квантовой статистике есть множество точек некоторой решетки на сфере данного радиуса, поэтому статистика на разбиениях должна учитывать кратность энергий частиц (см. далее). Статистика (мера) на $\mathcal{P}_{n}$ или $\mathcal{P}_{n, N}$ выбирается в соответствии с задачей, ее носитель может составлять часть множества $\mathcal{P}_{n}$, например $\mathcal{P}_{n, N(n)}$. Скейлинг сильно зависит от статистики и определяет соотношение между энергией всей конфигурации и числом частиц, энергия которых больше некоторого числа. Предельная конфигурация в эргодическом случае как элемент компакта $\mathcal{D}$ есть предельное распределение энергий частич, позволяюшее делать заключения того типа, что приведены выше: какая предельная доля общей энергии приходится на частицы с данными энергиями.

С точки зрения статистической физики мы рассматриваем систему в единичном объеме. Рост объема в нашей модели означает, что рассматриваются разбиения на нецелые слагаемые, точнее - на кратные некоторой обратной степени объема (степень зависит от размерности задачи). Но изменением масштаба мы приводим такое разбиение к обычному (иначе говоря, объем - к единичному). Однако в этом случае нужно пересчитать число слагаемых в новой шкале. Таким образом, обычный термодинамический предел укладывается в нашу схему со специальными мерами на $\mathcal{P}_{n, N}(n)$ и некоторой определяемой размерностью задачи зависимостью $N=N(n)$ (см. далее). Случай векторных разбиений отвечает ситуации, в которой кроме энергии имеются и другие первые интегралы.

\section{§2. Мультипликативные статистики. Большой канонический ансамбль}

Мы введем класс мер на $\mathcal{P}_{n}$, а затем покажем, что этот класс получается в качестве ограничения на $\mathcal{P}_{n}$ мер на $\mathcal{P}$, которые мы называем мультипликативньми.

Пусть задана последовательность функций $s=\left\{s_{k}\right\}, s_{k}: \mathbb{N} \rightarrow \mathbb{R}_{+}, k=1, \ldots$; зададим мультипликативную функцию разбиений $F_{s}(\lambda) \equiv F(\lambda)=\prod_{k} s_{k}\left(r_{k}(\lambda)\right)$, где $r_{k}=\#\left\{i: \lambda_{i}=k\right\}$ - числа заполнений. Пусть $Q_{n}=\sum_{\lambda \vdash n} F(\lambda)-$ статистическая сумма; обозначим через $\mu^{s, n} \equiv \mu^{n}$ следующую меру на $\mathcal{P}_{n}$ :

$$
\mu^{n}\left\{\lambda: r_{k}(\lambda)=r\right\}=Q_{n}^{-1} s_{k}(r), \quad \mu^{n}(\lambda)=Q_{n}^{-1} F(\lambda) .
$$

Аналогичным образом,

$$
\text { если } \quad Q_{n, N}=\sum_{\substack{\lambda \vdash n \\ N(\lambda)=N}} F(\lambda), \quad \text { то } \mu^{n, N}(\lambda)=Q_{n, N}^{-1} F(\lambda) .
$$

Мы определили класс мер на каноническом и малом каноническом ансамблях. Рассмотрим теперь большой канонический ансамбль - множество всех разбиений - и определим на нем семейства мер $\mu_{s, x}=\mu_{x}$, зависящих от вещественного параметра (параметров), также определяемых по семейству $s=\left\{s_{k}\right\}_{k=1}^{\infty}$,

$$
\mu_{x}(\lambda)=x^{n(\lambda)} \mathcal{F}(x)^{-1} \prod s_{k}\left(r_{k}(\lambda)\right)=x^{n(\lambda)} \mathcal{F}(x)^{-1} F(\lambda),
$$


где $\mathcal{F}(x)=\sum_{n=0}^{\infty} Q_{n} x^{n}$ (большая статсумма). Пусть последний ряд сходится при $0 \leqslant x<x_{0}$.

Заметим, что если положить $\mathcal{F}_{k}(y)=\sum_{r=0}^{\infty} s_{k}(r) y^{r}$, то

$$
\begin{aligned}
\mathcal{F}(x) & =\sum_{n=0}^{\infty} \sum_{\left\{r_{k}\right\}} \prod_{k} s_{k}\left(r_{k}\right) x^{n}=\sum_{n=0}^{\infty} \sum_{\left\{r_{k}\right\}} \prod s_{k}\left(r_{k}\right) x^{\sum k r_{k}} \\
& =\prod_{k=1}^{\infty} \sum_{r=0}^{\infty} s_{k}(r) x^{r_{k}}=\prod_{k=1}^{\infty} \mathcal{F}_{k}\left(x^{k}\right) .
\end{aligned}
$$

Лемма 2.1. Меры $\mu_{s, x} \equiv \mu_{x}$ определень при $x \in\left[0, x_{0}\right)$ и обладают свойствами

1) $\left.\mu_{x}\right|_{\mathcal{P}_{n}}(\lambda) \equiv \frac{\mu_{x}(\lambda)}{\mu_{x}\left(\mathcal{P}_{n}\right)}=\mu^{n}(\lambda)$

2) числа заполнения $r_{1}, \ldots$ как функции на $\left(\mathcal{P}, \mu_{x}\right)$ образуют последовательность независимых случайных величин относительно мер $\mu_{x}, x \in$ $\left[0, x_{0}\right)$;

3) $\mu_{x}=\sum_{n=0}^{\infty} \mathcal{F}(x)^{-1} x^{n} Q_{n} \mu^{n}$, т.е. $\mu_{x}$ есть выпуклал комбиначия мер $\mu^{n}$.

Все утверждения проверяются непосредственно. Семейства (по $x$ ) мер $\mu_{x}$, определенные выше, будем называть мультипликативными семействами. Для их задания достаточно задать производящую функцию $\mathcal{F}(x)$ вместе с ее разложением $\mathcal{F}(x)=\prod_{k=1}^{\infty} F_{k}\left(x^{k}\right)$. Многие интересные меры на разбиениях, например, мера Планшереля на диаграммах Юнга, не входят в этот класс, но, как мы увидим, среди мультипликативных семейств находятся важнейшие примеры.

Для того чтобы охватить общий случай, включающий фиксацию числа слагаемых, введем функцию двух переменных $\mathcal{F}(z, x)=\prod_{k=1}^{\infty} \mathcal{F}_{k}\left(z x^{k}\right)$. В этом случае

Положив, как и выше,

$$
\mathcal{F}(z, x)=\prod_{k=1}^{\infty} \sum_{r=0}^{\infty} s_{k}(r) z^{r} x^{k r}
$$

получаем

$$
Q_{n, N}=\sum_{\substack{n(\lambda)=n \\ N(\lambda)=N}} F_{s}(\lambda), \quad F_{s}(\lambda)=\prod_{k} s_{k}\left(r_{k}(\lambda)\right)
$$

$$
\mathcal{F}(z, x)=\sum_{n, N} Q_{n, N} x^{n} z^{N} .
$$

Введем семейство мер $\mu_{x, z}$ следующей формулой:

$$
\mu_{x, z}(\lambda)=x^{n(\lambda)} z^{N(\lambda)} \mathcal{F}(z, x)^{-1} F(\lambda) .
$$

Как и для семейства $\mu_{x}$, имеет место

ЛЕмма 2.2.1) Меры $\mu_{x, z}$ определень в вешественной области сходимости ряда для $\mathcal{F}(z, x), \mu_{x, 1}=\mu_{x}$. 
2) Ограничения мер $\mu_{x, z}$ на мальй канонический ансамбль суть мерь $\mu^{n, N}$ (см. вылие).

3) $\mu_{x, z}=\sum_{n, N} \mathcal{F}(z, x)^{-1} x^{n} z^{N} Q_{n, N} \mu^{n, N}$.

$B$ то же время числа заполнения уже не являются независимыми относительно мер $\mu_{x, z}$.

Рассмотрим теперь векторные разбиения. Разбиение вектора с натуральными координатами $\mathbf{n}=\left(n_{1}, \ldots, n_{d}\right)$ есть представление его в виде суммы неупорядоченных векторов

$$
\mathbf{n}=\mathbf{n}^{1}+\cdots+\mathbf{n}^{k}, \quad \mathbf{n}^{i}=\left(n_{1}^{i}, \ldots, n_{d}^{i}\right)
$$

Множество всех векторных разбиений $d$-вектора $\mathbf{n}$ обозначим через $\mathcal{P}_{\mathbf{n}}^{d}$, $\mathbf{n} \in \mathbb{Z}_{+}^{d}$, а множество разбиений с числом слагаемых $N$ - через $\mathcal{P}_{\mathbf{n}, N}^{d} \cdot$ Пусть

$$
\mathcal{P}^{d}=\bigcup_{\mathbf{n} \in \mathbb{Z}_{+}^{d}} \mathcal{P}_{\mathbf{n}}^{d}
$$

Аналогом чисел заполнения служит $d$-мерная матрица: пусть

$$
\begin{gathered}
\boldsymbol{\lambda} \in \mathcal{P}_{\mathbf{n}}^{d}, \quad \boldsymbol{\lambda}=\left(\mathbf{n}^{1}, \ldots, \mathbf{n}^{k}\right), \quad \mathbf{n}^{i} \in \mathbb{Z}_{+}^{d}, \quad \mathbf{n}=\sum_{i} \mathbf{n}^{i}, \\
r_{k_{1}, \ldots, k_{d}}(\boldsymbol{\lambda})=\#\left\{i: \mathbf{n}^{i}=\left(k_{1}, \ldots, k_{d}\right)\right\}
\end{gathered}
$$

а отображение $\varphi_{\lambda}$ из $\S 1$ заменяется на отображение

$$
\varphi_{\boldsymbol{\lambda}}^{d}\left(t_{1}, \ldots, t_{d}\right)=\sum_{\left(k_{1}, \ldots, k_{d}\right) \geqslant\left(t_{1}, \ldots, t_{d}\right)} r_{k_{1}, \ldots, k_{d}}(\boldsymbol{\lambda}) .
$$

Остается описать аналог отображения $\tau$ и компакта $\mathcal{D}$. Скейлинг в этом случае определяется $d$ нормирующими последовательностями $\left\{a_{n_{j}}^{j}: j=1, \ldots, d\right\}$, $n_{j} \in \mathbb{N}$, и

$$
\widetilde{\varphi}_{\boldsymbol{\lambda}}^{d}\left(t_{1}, \ldots, t_{d}\right)=\frac{1}{n_{1} \ldots n_{d}} a_{n_{1}}^{1} \ldots a_{n_{d}}^{d} \varphi\left(a_{n_{1}}^{1} t_{1}, \ldots, a_{n_{d}}^{d} t_{d}\right) .
$$

Компакт $\mathcal{D}^{d}$ строится аналогично: его элементы суть совокупности монотонно невозрастающих по всем аргументам функщий, заданных на координатных октантах и интегрируемых по лебеговой мере, и зарядов на бесконечностях. Проще всего описать $\mathcal{D}^{d}$ как пополнение множества невозрастающих плотностей, заданных во внутренности $\mathbb{R}_{+}^{d}$, по топологии равномерной сходимости на компактах, лежащих во внутренности $\stackrel{\circ}{\mathbb{R}_{+}^{d}}$. Отображение

$$
\tau^{d}: \mathcal{P}^{d} \rightarrow \mathcal{D}^{d}
$$

позволяет сформулировать те же вопросы о слабой сходимости образов мер, заданных на $\mathcal{P}^{d}$.

ЗАмечАнИЯ. 1. Подграфик функции $\varphi_{\boldsymbol{\lambda}}^{d}\left(t_{1}, \ldots, t_{d}\right)$ есть многомерное разбиение (многомерная диаграмма Юнга), однако не всякая диаграмма получается таким образом. 
2. При $d=2$ есть иной способ геометризации векторных разбиений, использованный в $[8,9]$, а именно упорядочение векторов-слагаемых $\mathbf{n}^{i}=\left(k_{1}^{i}, k_{2}^{i}\right)$ по возрастанию отношений $k_{1}^{i} / k_{2}^{i}$.

Мультипликативные семейства, заданные на $\mathcal{P}^{d}$, определяются также с помощью производящих функций

$$
\mathcal{F}\left(x_{1}, \ldots, x_{d}\right)=\prod_{k_{1}, \ldots, k_{d}} \mathcal{F}_{k_{1}, \ldots, k_{d}}\left(x_{1}^{k_{1}} \cdots x_{d}^{k_{d}}\right),
$$

а функция одного аргумента

$$
\mathcal{F}_{k_{1}, \ldots, k_{d}}(y)=\sum_{r=0}^{\infty} s_{k_{1}, \ldots, k_{d}}(r) y^{r}
$$

есть производящая функщия распределения числа заполнения $r_{k_{1}, \ldots, k_{d}}$. Если $\boldsymbol{\lambda} \in \mathcal{P}_{\mathbf{n}}^{d}, \mathbf{n}=\left(n_{1}, \ldots, n_{d}\right)$, то мера $\mu_{x_{1}, \ldots, x_{d}}$ задается так:

$$
\mu_{x_{1}, \ldots, x_{d}}(\boldsymbol{\lambda})=x_{1}^{n_{1}} \cdots x_{d}^{n_{d}} \mathcal{F}\left(x_{1}, \ldots, x_{d}\right)^{-1} \prod_{k_{1}, \ldots, k_{d}} s_{k_{1}, \ldots, k_{d}}\left(r_{k_{1}, \ldots, k_{d}}(\boldsymbol{\lambda})\right) .
$$

Меры $\mu_{x_{1}, \ldots, x_{d}}$ заданы для вещественных $x$ из области сходимости ряда для $\mathcal{F}\left(x_{1}, \ldots, x_{d}\right)$, причем числа заполнения независимы относительно этих мер. Мы не будем останавливаться на подробностях, отметим лишь, что формулы для мер на малом ансамбле $\mathcal{P}_{\mathbf{n}, N}^{d}$ задаются точно так же, как и для $d=1$.

\section{§3. Примеры мультипликативных мер}

Начнем с серии примеров алгебраического и комбинаторного происхождения. В них меры сначала задаются на естественных объектах — симметрической группе, разбиениях множества и т.п.; затем они (меры) переводятся в пространства $\mathcal{P}_{n}$ при всех $n$. Мультипликативность позволяет построить меры в большом каноническом ансамбле.

С другой стороны, в примерах физического характера столь же естественно определять сначала меры $\mu_{x}$ с помощью производящих функций, имеющих вид эйлеровых произведений или факторизованных (разложенных в бесконечное произведение) функций, а затем индуцировать эти меры на малый ансамбль. Мы используем обозначения $\S 2$.

1. Равномерная статистика на $\mathcal{P}_{\boldsymbol{n}}$ (аналог Бозе-статистики). Определим $\mu^{n}(\lambda)=p(n)^{-1}$ - равномерное распределение на $\mathcal{P}_{n}$. Соответствующая мультипликативная функция $F(\lambda)$ тождественно равна 1 и $s_{k}(r) \equiv 1$ при всех $k=1, \ldots$. Таким образом, $\mathcal{F}_{k}(x)=1 /(1-x)$,

$$
\begin{gathered}
\mathcal{F}(x)=\prod_{k=1}^{\infty} \mathcal{F}_{k}\left(x^{k}\right)=\prod_{k=1}^{\infty} \frac{1}{1-x^{k}} \\
\mu_{x}(\lambda)=x^{n(\lambda)} \mathcal{F}(x)^{-1}=x^{n(\lambda)} \prod_{k=1}^{\infty}\left(1-x^{k}\right), \quad \mu_{x}\left\{\lambda: r_{k}(\lambda)=s\right\}=x^{k s}\left(1-x^{k}\right) .
\end{gathered}
$$


Как мы увидим, эта статистика есть не что иное, как статистика двумерного Бозе-газа (см. [5]); она наиболее естественна и с теоретико-числовой точки зрения - все разбиения числа $n$ равновероятны и статсумма есть функция разбиений: $Q_{n}=p(n)$

1а. Если же мы рассматриваем фиксированное число слагаемых, то производящая функция такова:

$$
\mathcal{F}(z, x)=\prod_{k=0}^{\infty} \frac{1}{1-z x^{k}}=\sum_{n, N} p_{N}(n) x^{n} z^{N} .
$$

Здесь мера $\mu^{n, N}$ есть равномерное распределение на разбиениях числа $n$ с $N$ слагаемыми; при этом допускаются нулевые слагаемые и

$$
\begin{gathered}
\mathcal{F}_{k}(z, x)=\frac{1}{1-z x}, \quad \mu_{x, z}(\lambda)=x^{n(\lambda)} z^{N(\lambda)} \prod_{k=1}^{\infty}\left(1-z x^{k}\right), \\
\mu_{x, z}\left(\lambda: r_{k}(\lambda)=r, N(\lambda)=N\right)=x^{k r} z^{N}\left(1-z x^{k}\right) .
\end{gathered}
$$

Сомножитель $1 /(1-z)$ в п. 1а отвечает за распределение числа нулевых слагаемых, что существенно для объяснения эффекта конденсации Бозе-газа.

2. Равномерное распределение на разбиениях с различными слагаемыми (аналог Ферми-статистики). Тогда

$$
\begin{gathered}
\mathcal{F}(x)=\prod_{k=1}^{\infty}\left(1+x^{k}\right)=\sum_{n=0}^{\infty} p_{\neq}(n) x^{n}, \\
\mu^{n}(\lambda)=p_{\neq}(n)^{-1}, \quad \mu_{x}(\lambda)=x^{n(\lambda)} \prod_{k=1}^{\infty}\left(1+x^{k}\right)^{-1} .
\end{gathered}
$$

2а. В случае фиксации числа слагаемых имеем

$$
\begin{gathered}
\mathcal{F}(z, x)=\prod_{k=1}^{\infty}\left(1+z x^{k}\right)=\sum_{n, N} p_{\neq}(n, N) x^{n} z^{N}, \\
\mu^{n, N}(\lambda)=p_{\neq}(n, N)^{-1}, \quad \mu_{z, x}(\lambda)=x^{n(\lambda)} z^{N} \prod_{k=0}^{\infty}\left(1+z x^{k}\right)^{-1} .
\end{gathered}
$$

Следуя традициям теории разбиений, можно было бы по аналогии рассмотреть различные классы разбиений (например, с данной нижней границей разностей между слагаемыми, разбиения со слагаемыми из данного подмножества натуральных чисел и др.) с равномерной статистикой, но мы здесь ограничимся этими случаями и обобщим их в ином направлении.

3. Статистика Белла (см. $[11,12]$ ). Как правило, разбиения появляются как классы каких-либо объектов по некоторому отношению эквивалентности. Пусть $\Pi_{n}$ - совокупность всех разбиений множества из $n$ предметов; пусть $m_{n}$ - равномерное распределение на $\Pi_{n}$ и $\pi_{n}: \Pi_{n} \rightarrow \mathcal{P}_{n}$ - отображение, 
сопоставляющее разбиению $\Lambda \in \Pi_{n}$ разбиение $\lambda$ числа $n$, где $\lambda=\left(\lambda_{i}\right)$ и $\lambda_{i}$ есть мощности блоков разбиения $\Lambda$. Образ $\pi_{n} m_{n}=\beta_{n}$ есть мера на $\mathcal{P}_{n}$, называемая статистикой Белла (ср. числа Белла). Таким образом, это есть статистика на классах эквивалентности по действию симметрической группы. Очевидно, что

$$
\beta_{n}\{\lambda\}=1 / \prod_{k} r_{k}(\lambda) !(k !)^{r_{k}(\lambda)}, \quad \lambda \in \mathcal{P}_{n} .
$$

Меры $\beta_{n}$ являются мультипликативными, и соответствующая производящая функция есть

$$
\mathcal{F}_{k}(y)=e^{y / k !}, \quad \mathcal{F}(x)=\prod \mathcal{F}_{k}\left(x^{k}\right)=e^{e^{x}-1}, \quad s_{k}(r)=\frac{1}{(k !)^{r} r !} .
$$

Эта мера подробно исследована в работе [12].

Фиксация числа блоков дает производящую функцию

$$
\mathcal{F}(z, x)=e^{z e^{x}} .
$$

4. Статистика Хаара и меры Пуассона-Дирихле [11, 18]. Здесь рассматриваются равномерная мера $m_{n}$ на группе подстановок и ее деформация $m_{n}^{\theta}, \theta \in \mathbb{R}_{+}$:

$$
m_{n}^{\theta}(g)=\frac{1}{(n+\theta-1) \cdots \theta} \theta^{c(g)}=\frac{\theta^{c(g)}}{[\theta]^{n}}, \quad[\theta]^{n}=\theta(\theta+1) \cdots(\theta+n-1),
$$

где $c(g)$ — число циклов в подстановке $g \in S_{n}$. При $\theta=1$ мы получаем равномерное распределение на $S_{n}$.

Проекция $\pi: S_{n} \rightarrow \mathcal{P}_{n}$, сопоставляющая подстановке набор длин ее циклов, дает следующую меру $\mu^{\theta}$ на $\mathcal{P}_{n}$ :

$$
\mu^{\theta}(\lambda)=\frac{\theta^{N(\lambda)}}{\prod_{k} r_{k}(\lambda) ! k^{r_{k}(\lambda)}[\theta]^{n(\lambda)}}, \quad \theta \in[0, \infty) .
$$

При $\theta=1$ получаем распределение на разбиениях, называемое хааровским, так как оно индуцировано мерой Хаара на $S_{n}$. Все эти меры индуцированы мультипликативными мерами на $\mathcal{P}$; большая статсумма такова:

$$
\mathcal{F}(x)=\frac{1}{(1-x)^{\theta}}=e^{-\theta \ln (1-x)}=\prod_{k=1}^{\infty} e^{\theta x^{k} / k}=\prod_{k=1}^{\infty} \sum_{n=0}^{\infty} \frac{1}{n !}\left(\frac{\theta x^{k}}{k}\right)^{n},
$$

т. е. $\mathcal{F}_{k}(y)=e^{\theta y / k}$. Учет числа циклов дает

$$
\mathcal{F}_{k}(z y)=e^{\theta z y / k}, \quad \mathcal{F}(x, z)=\prod_{k=1}^{\infty} \mathcal{F}_{k}\left(z x^{k}\right)=\frac{1}{(1-x)^{z \theta}},
$$

т.е. $\theta$ играет роль переменной $z$; таким образом, можно положить $\mathcal{F}(x, z)=$ $1 /(1-x)^{z}$.

5. Модель $\boldsymbol{d}$-мерного идеального газа [5]. Рассмотрим более близкую к квантовой статистической физике модель. Мы будем ограничиваться лишь указанием большой статсуммы (т.е. производящей функции), все остальные компоненты легко восстанавливаются. Напомним, что для идеального газа энергия 
системы есть сумма $E=\sum_{\mathbf{p}} r_{\mathbf{p}} \varepsilon_{\mathbf{p}}$, где $\varepsilon_{\mathbf{p}}$ - энергия частицы с импульсом $\mathbf{p} \in \mathbb{R}^{d}$, т. e.

$$
\varepsilon_{\mathbf{p}}=\frac{1}{2 m}\|\mathbf{p}\|^{2}=\frac{1}{2 m}\left(\frac{2 \pi \hbar}{V^{1 / d}}\right)^{2}\|\mathbf{q}\|^{2}
$$

(т.е. $\varepsilon_{\mathbf{p}}$ - собственные числа оператора Лапласа в евклидовом торе) (см. [5]), $\mathbf{q} \in \mathbb{Z}^{d}, V$ - объем, $\hbar$ - постоянная Планка, $m$ - масса, $d$ - размерность, $r_{\mathbf{p}}$ - число частиц с импульсом $\mathbf{p},\|\mathbf{q}\|^{2}=q_{1}^{2}+\cdots+q_{d}^{2}$. Примем множитель при $\|\mathbf{q}\|^{2}$ за единицу (о случае растущего объема см. замечание в конце $\S 1$ ).

Переходя к нашим обозначениям, мы получаем разбиение числа $n(=E)$ со слагаемыми, являющимися суммами $d$ квадратов натуральных чисел, причем слагаемые считаются различными, т.е. параметром слагаемого служит вектор из $\mathbb{Z}^{d}$, а самим слагаемым - квадрат его нормы. Это приводит к следующей производящей функции для Бозе-статистики:

$$
\mathcal{F}(x)=\prod_{k=1}^{\infty} \frac{1}{\left(1-x^{k}\right)^{j_{d}(k)}}
$$

где $j_{d}(k)$ - число представлений числа $k$ суммой $d$ квадратов. Напомним, что

$$
\sum_{k=0}^{\infty} j_{d}(k) z^{k}=\theta(0, \tau)^{d}
$$

где $\theta(0, \tau)=\sum_{n \in \mathbb{Z}} e^{\pi i \tau n^{2}}-$ тэта-функция.

Для Ферми-статистики получаем

$$
\mathcal{F}(x)=\prod_{k=1}^{\infty}\left(1+x^{k}\right)^{j_{d}(k)} .
$$

Если же учитывается число частиц или слагаемых, то соответствующие функции выглядят так:

$$
\mathcal{F}_{b}(z, x)=\prod \frac{1}{\left(1-z x^{k}\right)^{j_{d}(k)}}, \quad \mathcal{F}_{f}(z, x)=\prod\left(1+z x^{k}\right)^{j_{d}(k)} .
$$

Все эти функции определяют по схеме $\S 2$ меры $\mu^{n}$ на малом и $\mu_{x}$ на большом канонических ансамблях; меры $\mu_{x}$ и $\mu_{x, z}$ являются мультипликативными и к ним применим метод нахождения предельной конфигурации.

6. Классические производящие функции. Предыдущие примеры охватываются общей функцией вида

$$
\mathcal{F}(x)=\prod_{k=1}^{\infty} \frac{1}{\left(1-x^{k}\right)^{b_{k}}} \quad \text { или } \quad \mathcal{F}(x)=\prod_{k=1}^{\infty}\left(1+x^{k}\right)^{b_{k}},
$$

где $\left\{b_{k}\right\}$ - произвольная последовательность натуральных чисел. Соответствующее распределение на $\mathcal{P}_{n}$ можно трактовать следующим образом: считаем, что имеется $b_{k}$ типов слагаемых, равных $k$ (частиц с энергией $k$ ) и все разбиения 
различаются по составу и по типу слагаемых; само же распределение равномерно на всех таких разбиениях. Для дальнейшего, так же как и для вычисления логарифмической асимптотики коэффициентов функции $\mathcal{F}(x)$ (см. теорему Мейнардуса в [13]), существенна лишь следующая константа $\alpha$, определяемая последовательностью $\left\{b_{k}\right\}$ : пусть $\sum_{k=1}^{\infty} b_{k} / k^{s}$ - ряд Дирихле, который сходится при всех $s, \operatorname{Re} s>\alpha>0$. Заметим, что $\alpha=1$ для примера $1\left(b_{k} \equiv 1\right), \alpha=1 / 2$ в примере 5 для $d=1\left(j_{1}(k)=1\right.$, если $k$ - полный квадрат, и 0 в остальных случаях). По известной теореме в общем случае (см. Зигель [14]) $\alpha=d / 2$ для $j_{d}(\cdot)$.

Таким образом, для $d$-мерной модели идеального газа производящая функция может быть заменена на

$$
\prod \frac{1}{\left(1-z x^{k}\right)^{\left[k^{\beta(d)}\right]}}, \quad \text { где } \beta(d)=\frac{d-2}{2}, \alpha=\beta+1, d \geqslant 2 .
$$

В частности, основной для нас случай $\prod 1 /\left(1-x^{k}\right)$ соответствует $\beta(d)=0$, т. е. $d=2$.

7. Фиксированный размер разбиений. Мы лишь упомянем равномерные распределения на разбиениях с фиксированным ростом числа слагаемых и их размеров. В этом случае соответствуюшие диаграммы содержатся в прямоугольнике с заданным ростом длин сторон, а вместо одной мультипликативной меры здесь следует рассмотреть последовательность таких мер. Такая же ситуация в случае квазибольцмановской статистики (см. [4]). Один пример приводится далее.

8. Векторные разбиения. Пусть $\mathcal{P}_{\mathbf{n}}^{d}, d \in \mathbb{N}, \mathbf{n} \in \mathbb{Z}_{+}^{d}$, - множество всех разбиений вектора $\mathbf{n}=\left(n^{1}, \ldots, n^{d}\right)$ в сумму неупорядоченных слагаемых с натуральными координатами и $\mathcal{P}^{d}=\bigcup \mathcal{P}_{\mathbf{n}}^{d}$.

Производящая функция

$$
\mathcal{F}\left(x_{1}, \ldots, x_{d}\right)=\prod_{\left(k_{1}, \ldots, k_{d}\right)} \frac{1}{1-x_{1}^{k_{1}} \cdots x_{d}^{k_{d}}}=\sum_{\mathbf{n} \in \mathbb{Z}_{+}^{d}} p(\mathbf{n}) x^{\mathbf{n}}
$$

отвечает мультипликативной мере, ограничение которой на $\mathcal{P}_{\mathbf{n}}^{d}$ есть равномерная мера на всех разбиениях.

Можно обобщить на $d$-мерный случай и другие мультипликативные меры, например

$$
\mathcal{F}(x)=\prod_{\mathbf{k} \in \mathbb{Z}_{+}^{d}}\left(1+x^{\mathbf{k}}\right) \quad \text { и т.д. }
$$

Особый класс составляют строгие разбиения (произведение берется по наборам векторов $\left(k_{1}, \ldots, k_{d}\right) \in \mathbb{Z}_{+}^{d}$ с взаимно простыми (в совокупности) координатами, см. [8]). Напомним, что для $d=2$ есть две различные геометрические интерпретации векторных разбиений (см. §2). Как уже говорилось, векторные разбиения отвечают в статистической физике системам с дополнительными первыми интегралами. 


\section{§4. Основные результаты}

1. Слабая эквивалентность ансамблей. Рассмотрим здесь лишь обычные (одномерные) разбиения без фиксации числа частиц. Пусть $\mu_{x}$ - некоторая мультипликативная мера на $\mathcal{P}, x \in\left(0, x_{0}\right)$. В соответствии с $\S 1$ прежде всего нужно отыскать нормирующую последовательность $\left\{a_{n}\right\}=a$. После этого можно построить отображение $\tau_{a}: \mathcal{P} \rightarrow \mathcal{D}$.

Напомним, что $\mu_{x}=\sum_{n} \mathcal{F}(x)^{-1} Q_{n} x^{n} \mu^{n}$.

ТЕОРема 4.1 (скейлинг). Нормировка $\left\{a_{n}\right\}$ для мультипликативных мер, порожденных производящей функиией

$$
\mathcal{F}(x)=1 / \prod_{k}\left(1-x^{k}\right)^{\left[k^{\beta}\right]}, \quad 0<x<1, \beta \geqslant 0,
$$

при которой существует нетривиальный предел мер $\lim _{x \rightarrow 1} \tau_{a}^{*} \mu_{x}$ в пространстве $\mathcal{D}$ обобщенных диаграмм, такова:

$$
a_{n}=n^{1 /(2+\beta)} .
$$

В частности, для $\beta=0$ имеем $a_{n}=\sqrt{n}$.

Та же нормировка справедлива для $\mathcal{F}(x)=\prod\left(1+x^{k}\right)^{\left[k^{\beta}\right]}$.

ТЕОРема 4.2 (вырожденность предела). При нормировке из теоремь 4.1 предел мер $\lim _{x \rightarrow 1} \tau_{a} \mu_{x}$ существует и является вырожденной мерой, сосредоточенной на одной непрерьвной кривой.

ТЕорема 4.3 (слабая эквивалентность ансамблей). В условиях теоремы 4.1 имеет место слабал эквивалентность ансамблей, т.е.

$$
\lim _{x \rightarrow 1} \tau^{*} \mu_{x}=\lim _{n \rightarrow \infty} \tau^{*} \mu^{n} .
$$

\section{2. Явные ответы.}

ТЕОРемА 4.4 (о предельной конфигурации для обобщенной одномерной Бозестатистики). Пусть

$$
\mathcal{F}(x)=\prod_{k=1}^{\infty} \frac{1}{\left(1-x^{k}\right)^{\left[k^{\beta}\right]}}
$$

- производящая функиия и $\mu_{x}$ - мультипликативная мера, отвечаюшая ей. Пусть меры $\mu^{n}$ есть индуцированнье мерами $\mu_{x}$ меры на $\mathcal{P}_{n}$. (При $\beta=0$ $\mu^{n}$ есть равномерное распределение на $\left.\mathcal{P}_{n}.\right)$ Определим отображение $\tau_{a}: \mathcal{P}_{n} \rightarrow \mathcal{D}$ для скейлинга $a=\left\{a_{n}\right\}, a_{n}=n^{1 /(2+\beta)}$, и функции

$$
\widetilde{\varphi}_{\lambda}(t)=n^{-(1+\beta) /(2+\beta)} \sum_{k \geqslant t n^{1 /(2+\beta)}} r_{\lambda}(k)
$$

(напомним, что $r_{k}(\lambda)$ - иисло слагаемьх, равньх $k$, в разбиении $\lambda$ ).

Тогда для всяких $\varepsilon>0,0<a, b<\infty$ суиествует такое $n_{0}$, ито при $n>n_{0}$

$$
\mu^{n}\left\{\lambda \in \mathcal{P}_{n}: \sup \left|\widetilde{\varphi}_{\lambda}(t)-C_{\beta}(t)\right|<\varepsilon\right\}>1-\varepsilon,
$$


где $C_{\beta}(t)$ - функчия (плотность распределения), задаваемая формулой

$$
C_{\beta}(t)=\int_{t}^{\infty} u^{\beta} \frac{e^{-c u}}{1-e^{-c u}} d u,
$$

$c$ - константа, определяемая условием $\int_{0}^{\infty} C_{\beta}(t) d t=1$.

В частности, при $\beta=0$ имеем $C_{\beta}(t)=-(\sqrt{6} / \pi) \ln \left(1-e^{(\pi / \sqrt{6}) t}\right), c=$ $\sqrt{\zeta(2)}$, или, в более симметричной форме,

$$
e^{-(\pi / \sqrt{6}) x}+e^{-(\pi / \sqrt{6}) y}=1 .
$$

Формула (**) выведена автором сначала с помощью некоторых формул из [15] (см. [16, 19]), позже она получена им иным, более естественным, способом, обобщенным на широкий круг задач в данной статье.

При $\beta=1$

$$
C_{1}(t)=-t \ln \left(1-e^{-c t}\right)+c^{-1} \operatorname{Li}_{2}\left(e^{-c t}\right),
$$

где $\mathrm{Li}_{2}$ - дилогарифм

$$
\operatorname{Li}_{2}(u)=\sum_{n=1}^{\infty} \frac{u^{n}}{n^{2}} .
$$

Функция $C_{\beta}(t)$ может быть выражена через ряд от неполной $Г$-функции $[17]$ :

$$
\begin{aligned}
C_{\beta}(t) & =\int_{t}^{\infty} \frac{u^{\beta} e^{-c u}}{1-t^{-c u}} d u=\sum_{k=1}^{\infty} \int_{t}^{\infty} u^{\beta} e^{-c k u} d u=\sum_{k=1}^{\infty} \frac{1}{c^{\beta} k^{\beta+1}} \int_{c k t}^{\infty} u^{\beta} e^{-u} d u \\
& =\frac{1}{c^{\beta}} \sum_{k=1}^{\infty} \frac{1}{k^{\beta+1}} \Gamma(\beta+1, c k t)=\frac{1}{c^{\beta}} \sum_{k=1}^{\infty} \frac{1}{k^{\alpha}} \Gamma(\alpha, c k t)
\end{aligned}
$$

$(\alpha=\beta+1=d / 2-$ простой полюс соответствующего ряда Дирихле, см. $\S 3)$.

ЗАмечания. 1. Мера $\mu^{n}$ на $\mathcal{P}_{n}$, отвечающая $\beta=(d-2) / 2, d \geqslant 2$, совпадает с Бозе-статистикой идеального газа в размерности $d$ (число частиц не фиксируется - «фотонный случай»). Таким образом, функция $C(t)$ в теореме 4 (формула $(*))$ дает плотность распределения энергии по спектру частищ; типичная энергия одной частицы имеет порядок $E^{2 /(d+2)}(E=n)$ и ограничена при всех $\beta>0(d>2)$.

2. Явный вид меры $\mu^{n}$ на $\mathcal{P}_{n}$ легко выписать через гауссовы коэффициенты.

3. Мультипликативные меры на микроканонических ансамблях $\mathcal{P}_{n, N}$ (разбиений с заданным числом слагаемых) получаются из функций

$$
\mathcal{F}(x)=\prod_{k=1}^{\infty}\left(1-z x^{k}\right)^{-\left[k^{\beta}\right]} .
$$

Термодинамическому пределу отвечает выбор роста

$$
N=N(n)=v n^{d /(d+2)}=v n^{1-2 /(2 \beta+3)} .
$$

Соответствующую предельную форму можно также получить нашими методами; при этом для $d=3$ обнаруживается естественное объяснение конденсации БозеЭйнштейна. Однако можно рассматривать и иной рост $N(n)$. 
Аналогичные результаты можно доказать для разбиений с неодинаковыми слагаемыми (Ферми-статистики). Пусть

$$
\mathcal{F}(x)=\prod_{k=1}^{\infty}\left(1+x^{k}\right)^{\left[k^{\beta}\right]} ;
$$

построим соответствующие меры $\mu_{x}$ и $\mu^{n}$ на большом и малом канонических ансамблях $\mathcal{P}, \mathcal{P}_{n}$. Тогда в тех же обозначениях, что и выше, ответ можно записать в тех же терминах.

ТЕОРЕмА 4.5 (о предельной конфигурации для обобщенной Ферми-статистики). Для всяких $\varepsilon>0,0<a, b<\infty$, существует такое $n_{0}$, что при $n>n_{0}$

$$
\mu^{n}\left\{\lambda \in \mathcal{P}_{n}: \sup _{t \in[a, b]}\left|\widetilde{\varphi}_{\lambda}(t)-C_{f}(t)\right|<\varepsilon\right\}>1-\varepsilon,
$$

где $C_{f}(t)$ - следующая функиия (плотность распределения):

$$
C_{f}(t)=\int_{t}^{\infty} u^{\beta} \frac{e^{-c u}}{1+e^{-c u}} d u
$$

причем константа с определяется условием

$$
\int_{0}^{\infty} C_{f}(t) d t=1
$$

в частности, дяя $\beta=0$ (равномерного распределения на разбиениях с неравньли членами)

$$
C_{f}(t)=(\sqrt{12} / \pi) \ln \left(1+e^{-(\pi / \sqrt{12}) t}\right) \quad \text { или } \quad e^{-(\pi / \sqrt{12}) y}-e^{-(\pi / \sqrt{12}) x}=1 .
$$

Рассмотрим квазибольцмановскую статистику (см. [5, 6])

$$
\mathcal{F}(x)=e^{x /(1-x)}=\prod_{k=1}^{\infty} e^{x^{k}}
$$

Она отвечает распределению на $\mathcal{P}$, задаваемому формулой для мультипликативной меры

$$
\mu_{x}\left\{\lambda: r_{k}(\lambda)=i\right\}=\frac{x^{k i}}{i !} e^{-x^{k}} .
$$

ТЕОРема 4.6. Предельная конфигураиия для случайньх разбиений, подчиненных статистике $\mu_{x}$, задается формулой

$$
\lim _{x \rightarrow 1} \mu_{x}\left\{\lambda: \sup _{0<t<\infty}\left|\widetilde{\varphi}_{\lambda}(t)-e^{-t}\right|<\varepsilon\right\}=1 .
$$

Та же кривая $C(t)=e^{-t}$ является предельной для мер $\mu^{n}$ (индуцированньх мерой $\mu_{x}$ на $\left.\mathcal{P}_{n}\right)$ nри $n \rightarrow \infty$. 
Еще один класс статистик возникает при ограничении роста размера слагаемых в разбиении и их числа. Рассмотрим функцию

$$
\mathcal{F}_{N}(x)=\prod_{1}^{\theta \sqrt{N}} \frac{1}{1-t x^{k}}=\sum_{n, m} q_{N}(n, m) t^{m} x^{n}
$$

и будем искать предельную конфигурацию при $n=N \rightarrow \infty$ при условии $m=$ $\rho \sqrt{N}$. Это означает, что соответствующее распределение равномерно на разбиениях числа $N$, у которых число слагаемых $\leqslant \rho \sqrt{N}$, а их величина $\leqslant \theta \sqrt{N}$. Соответствующая диаграмма лежит в прямоугольнике $\rho \sqrt{N} \times \theta \sqrt{N}, \rho \theta \geqslant 1$.

ТЕОРема 4.7. Предельная конфигурачия дается формулой

$$
\frac{1-e^{c \theta}}{1-e^{-c(\theta+\rho)}} e^{-c y}+\frac{1-e^{-c \rho}}{1-e^{-c(\theta+\rho)}} e^{-c x}=1 .
$$

При $\rho=\theta$ (квадрат) эта функиия имеет вид

$$
e^{-c y}+e^{-c x}=1-e^{-c \theta},
$$

где константа с ищется из условия $\int_{0}^{\infty} y d x=1$. При $\theta \rightarrow \infty$ она переходит в функцию из теоремь $4.4(\beta=0)$, формула $(* *)$.

Рассмотрим теперь векторные разбиения. Мы приведем самый простой пример - равномерного распределения $\mu^{\mathbf{n}}$ на векторных разбиениях $\mathcal{P}_{\mathbf{n}}^{d}$. Пусть

$$
\mathcal{F}(x)=\prod_{k} \frac{1}{1-\mathbf{x}^{k}}=\sum p_{d}(\mathbf{n}) x^{\mathbf{n}} ;
$$

здесь $\mathbf{x}=\left(x_{1}, \ldots, x_{d}\right), \mathbf{k}=\left(k_{1}, \ldots, k_{d}\right), \mathbf{n}=\left(n_{1}, \ldots, n_{d}\right), \mathbf{x}^{\mathbf{k}}=\prod_{i=1}^{d} x_{i}^{k_{i}}$. Определим скейлинг, ограничившись случаем $\mathbf{n}=(n, \ldots, n)$,

$$
\mathbb{R}_{+}^{d} \ni t=\left(t_{1}, \ldots, t_{d}\right) \rightarrow n^{(d+1)^{-1}} t \quad \text { и } \quad \widetilde{\varphi}_{\lambda}(t)=\frac{1}{n^{d /(d+1)}} \varphi\left(n^{(d+1)^{-1}} t\right) .
$$

Tеорема 4.8. Для всякого $\varepsilon>0, d \geqslant 2$

$$
\lim _{n \rightarrow \infty} \mu^{\mathbf{n}}\left\{\lambda: \sup _{t}\left|\widetilde{\varphi}_{\lambda}(t)-C_{d}(t)\right|<\varepsilon\right\}=1,
$$

¿de

$$
\begin{gathered}
C_{d}(t)=\gamma^{-d} \operatorname{Li}_{d}\left(\exp \left(-\gamma \sum_{i=1}^{d} t_{i}\right)\right), \\
\gamma=\zeta(d+1)^{(d+1)^{-1}}, \quad \operatorname{Li}_{d}(x)=\sum_{n=1}^{\infty} \frac{x^{n}}{n^{d}}, \quad d \geqslant 2 .
\end{gathered}
$$

ЗАмечАния. 1 . В отличие от $d=1$ плотность $C_{d}$ при $d=2$ ограничена в $\mathbb{R}_{+}^{d}$.

2 . При $d=2$ векторное разбиение $\left(n_{1}, n_{2}\right)=\sum_{i}\left(n_{1}^{i}, n_{2}^{i}\right)$ имеет другой геометрический образ - это выпуклая целочисленная ломаная, соединяющая точки $(0,0)$ и $\mathbf{n}=\left(n_{1}, n_{2}\right)$ - сумму, а именно, слагаемые можно упорядочить по 
возрастанию отношения $n_{1}^{i} / n_{2}^{i}$ и рассмотреть последовательные суммы. Соответствующая асимптотическая задача изучена в [8-10]; метод этой статьи дает уточнение этих результатов.

3. Подграфик функции $\varphi_{\lambda}$ есть $(d+1)$-мерная диаграмма Юнга, однако не произвольная, а удовлетворяющая некоторому условию положительности (так как $\varphi_{\lambda}$ есть функция распределения в определенном смысле). Таким образом, мы нашли предельную конфигурацию для равномерного распределения на специальных $d$-мерных диаграммах Юнга. Вопрос о предельной форме для равномерного распределения на множестве всех даже трехмерных диаграмм пока остается открытым.

\section{§5. Формулировки лемм}

Мы наметим план доказательства результатов из $§ 4$. Будем следовать обозначениям $\S \S 1-3$. Для простоты мы рассмотрим лишь обычные (а не векторные) разбиения и мультипликативные меры на $\mathcal{P}$ и $\mathcal{P}_{n}$ соответственно. Эти меры задаются производящей функщией, аналитической в круге $|x| \leqslant 1$ и разложенной в произведение

$$
\mathcal{F}(x)=\prod_{k} \mathcal{F}_{k}\left(x^{k}\right), \quad \mathcal{F}_{k}(y)=\sum_{r=0}^{\infty} s_{k}(r) y^{r}, \quad s_{k}(r) \geqslant 0 .
$$

Мера $\mu_{x}$ задается формулой (2.1), а $\mu^{n}$ - формулой (2.2). Связь между ними дается формулой (см. лемма 2.1)

$$
\mu_{x}=\sum_{n} x^{n} \mathcal{F}(x)^{-1} Q_{n} \mu^{n},
$$

где $Q_{n}$ - статсумма. Если выбран скейлинг $a=\left\{a_{n}\right\}$, то заданы отображения $\tau_{a}: \mathcal{P} \rightarrow \mathcal{D}$, и меры $\mu_{x}, \mu^{n}$ отображаются в $\tau_{a}^{*} \mu_{x}, \tau_{a}^{*} \mu^{n}$. Мы сохраним за ними те же обозначения $(\mu)$ без риска смешения, так как скейлинг выбирается однозначно.

Формула (5.1) показывает, что $\mu_{x}$ есть выпуклая комбинация мер $\mu^{n}$. Поэтому очевидно следующее утверждение.

ЛЕмма 5.1. Если существует слабый предел в $\mathcal{D}$ мер $\mu^{n}$, то тот же предел имеют мерьл $\mu_{x}$ при $x \rightarrow 1$.

Будем говорить, что имеет место эргодический случай (случай точки), если предельная мера для $\mu_{x}$ при $x \rightarrow 1$ существует и вырожденна, т. е. сосредоточена в одном элементе пространства $\mathcal{D}$ или является $\delta$-мерой; этот элемент мы и назовем предельной конфигурацией (формой, распределением) в данной задаче. Если же предел существует, но не является $\delta$-мерой, то будем говорить, что имеет место неэргодический случай. (Таков, например, случай хааровской статистики.)

Обратное утверждение требует уже «тауберовых» аргументов и является в нашей ситуации нетривиальным. 
Лемма 5.2. Если существует слабый предел в $\mathcal{D}$ мультипликативных мер $\mu_{x}$ при $x \rightarrow 1$ и предельная мера вырожденна, то тот же предел имеют меры $\mu^{n}$.

Доказательство существенно использует мультипликативность мер $\mu_{x}$ и носит характер тауберовой теоремы. Вопрос об общих тауберовых теоремах в этой ситуации (для неэргодического случая или для немультипликативных мер) представляет большой интерес и, видимо, не изучался. Теперь можно ограничиться изучением только лишь гиббсовских мер $\mu_{x}$, что ввиду мультипликативности сводит вопрос к задаче теории вероятностей (локальной предельной теореме). Лемма 2 есть по существу утверждение об эквивалентности большого и исходного канонических ансамблей.

В дальнейшем рассматриваются производящие функции вида

$$
\mathcal{F}(x)=\prod_{k=1}^{\infty}\left(1-x^{k}\right)^{-\left[k^{\beta}\right]}, \quad \mathcal{F}(x)=\prod_{k=1}^{\infty}\left(1+x^{k}\right)^{\left[k^{\beta}\right]}, \quad \beta \geqslant 0 .
$$

Случай функций нескольких аргументов или функций $\mathcal{F}(z, x)=\prod_{k}\left(1+z x^{k}\right)^{\left[k^{\beta}\right]}$ отличается лишь большей громоздкостью.

Найдем скейлинг для этих функций; он полностью определяется показателем $\beta$.

Лемма 5.3. Мультипликативная мера $\mu_{x}$, определенная функииями указанного вида и перенесенная на компакт $\mathcal{D}$ с помощью отображения $\tau_{a}^{*}$, где скейлинг $a=\left\{a_{n}\right\}$ имеет вид $a_{n}=n^{(2+\beta)^{-1}}$, имеет вырожденный слабый предел $C=C(\cdot) \in \mathcal{D}$, m.e.

$$
\lim _{x \rightarrow 1} \mu_{x}\left\{\lambda: \sup _{b_{1} \leqslant t \leqslant b_{2}}\left|\widetilde{\varphi}_{\lambda}(t)-C(t)\right|<\varepsilon\right\}=1,
$$

где $\left[b_{1}, b_{2}\right] \subset(0, \infty)$ - произвольный компакт в $\mathbb{R}_{+}$, т.е.

$$
\mathrm{w}_{x \rightarrow 1} \mu_{x}=\delta_{C},
$$

а $C(\cdot)$ - предельная конфигурачия.

Доказательство леммы 5.3 проходит одновременно с процедурой явного нахождения слабого предела. И именно здесь мы должны преодолеть ту же трудность, которая встречается при применении метода перевала. Собственно, достаточно найти среднее меры $\mu_{x}$ на $\mathcal{D}$ и оценить дисперсию при $x \rightarrow 1$. Однако для этого нужно выбрать $x$ так, чтобы можно было воспользоваться преимуществами мультипликативности (независимости). Рассмотрим выражение

$$
\begin{gathered}
E_{x} \widetilde{\varphi}_{\lambda}(t)=E_{x} \frac{a_{n}}{n} \sum_{k \geqslant t a_{n}} r_{k}(\lambda)=E_{x} n(\lambda)^{\gamma-1} \sum_{k \geqslant t n(\lambda)^{\gamma}} r_{k}(\lambda), \\
\gamma=(2+\beta)^{-1}, \quad E_{x} \equiv E_{\mu_{x}} .
\end{gathered}
$$

Поскольку множитель $n(\lambda)$ зависит от $\lambda$, то следует сначала подобрать $x=x_{n}$ так, чтобы $n(\lambda)$ имело заданное значение. 
ЛЕмма 5.4. Имеем

$$
E_{x} r_{k}(\lambda)=\left.y\left[\ln \mathcal{F}_{k}(y)\right]^{\prime}\right|_{y=x^{k}}, \quad E_{x} \sum_{k} k r_{k} \equiv E_{x} n(\lambda)=x[\ln \mathcal{F}(x)]^{\prime} .
$$

Уравнение (для $\mathcal{F}$ указанного вида)

$$
E_{x} n(\lambda)=x[\ln \mathcal{F}(x)]^{\prime}=n \in \mathbb{N}
$$

имеет единственное решение $x_{n} \in(0,1)$, и при этом

$$
\lim _{n \rightarrow \infty} E_{x}\left(\frac{n(\lambda)}{n}-1\right)^{2}=0 \text {. }
$$

Выбор $x_{n}$ и есть выбор перевального контура в методе перевала (или критическая точка в методе Лапласа и т. д.) для оценки статсумм. Преимущество вероятностного метода все же состоит в том, что вместо хлопотливых оценок в окрестности точки перевала достаточно оценить дисперсию (лемма 5.6 ниже).

Последняя лемма дает возможность заменить $n(\lambda)$ на $n$ в (5.2) и тем самым найти среднее $E_{x} \widetilde{\varphi}_{\lambda}(t)$, которое и дает значение предельной конфигурации в точке $t$.

Лемма 5.5. Пусть $\mathcal{F}(x)=\prod_{k=1}^{\infty}\left(1-x^{k}\right)^{-k^{\beta}} ;$ тогда

$$
E_{x_{n}} \widetilde{\varphi}_{\lambda}(t) \equiv C(t)=\int_{t}^{\infty} u^{\beta} \frac{e^{-c u}}{1-e^{-c u}} d u
$$

где с - некоторая константа.

Доказательство вытекает из того, что сумма

$$
E_{x_{n}} \widetilde{\varphi}_{\lambda}(t) \simeq \frac{1}{n^{1-\gamma}} \sum_{k \geqslant t n^{\gamma}}^{\prime} E_{x_{n}} r_{k}(\lambda)
$$

в (5.2) является интегральной суммой для правой части (5.3).

Для других функций, упомянутых выше, вычисления проводятся так же.

Заключительная лемма утверждает, что предельная мера вырожденна, т.е. является $\delta$-мерой, сосредоточенной в пределе средних $C(\cdot)$.

Лемма 5.6. $\lim _{n \rightarrow \infty} E_{x_{n}}\left[\widetilde{\varphi}_{\lambda}(t)-C(t)\right]^{2}=0$.

Реализация этого плана для более общих мер (даже мультипликативных) наталкивается на аналитические трудности, однако мы думаем, что приведенная схема адекватна во многих задачах асимптотической комбинаторики, приложениях и аддитивных задачах теории чисел, геометрических приложениях и, возможно, в задачах статфизики.

\section{§6. Вариационный принцип}

Возможен совершенно иной подход к задачам асимптотической комбинаторики и, в частности, к задаче о предельной форме, напоминающий одновременно и метод континуального интеграла, и принщип минимума действия, и метод больших уклонений в теории вероятностей. Предположим для определенности, что задана 
какая-либо мера $\mu^{n}$ на диаграммах Юнга, которые определены на последовательности решеток $\frac{1}{a_{n}} \mathbb{Z}_{+} \times \frac{a_{n}}{n} \mathbb{Z}_{+} \equiv L_{n}$, и $\Gamma$ - некоторый элемент из $\mathcal{D}$, лежаший в $\mathbb{R}_{+}^{2}$. Рассмотрим его $\varepsilon$-окрестность $U_{\varepsilon}(\Gamma)$ и вычислим $\mu^{n}\left\{\lambda: \lambda \in U_{\varepsilon}(\Gamma)\right\}$. При надлежащей нормировке $\gamma_{n}$ можно вычислить

$$
\lim _{\varepsilon \rightarrow 0} \lim _{n \rightarrow \infty} \frac{1}{n^{\gamma}} \ln \mu^{n}\left\{\lambda: \lambda \in U_{\varepsilon}(\Gamma)\right\} .
$$

При фиксированных мерах $\mu^{n}$ этот предел (если он существует) определяет функционал действия на кривых из $\mathcal{D}$. Естественно предположить, что предельные кривые для статистик $\left\{\mu^{n}\right\}$ при $n \rightarrow \infty$ (опять-таки, если они существуют) доставляют максимум этого функционала. Отдельный вопрос состоит в том, всегда ли совокупность максимумов этого функционала совпадает с запасом предельных кривых. Если это так, мы будем говорить, что выполнен вариационный принцип (для данной задачи). Значение $\max$ можно рассматривать как энтропию последовательности мер $\mu^{n}$, а значение функционала на произвольной кривой - как емкость кривой, см. [8]. Мы приведем здесь формулировку, показывающую, что в ситуациях, рассмотренных в $\S \S 3-5$, вариационный принцип выполнен. В то же время явное выражение действия иногда слишком громоздко.

Рассмотрим равномерное распределение на разбиениях (диаграммах) внутри квадрата; более точно, зафиксируем квадрат $[0,1]^{2}$ и рассмотрим множество $X_{n}$ всех ступенчатых монотонно невозрастающих функций $\varphi$ с $\varphi(0)=1, \varphi(1)=0$, со ступеньками, лежашими в узлах решетки $\left(\frac{1}{n} \mathbb{Z}\right)^{2}$, и с фиксированным интегралом $\int_{0}^{1} \varphi(t) d t=\rho, 0<\rho<1 / 2$, и равномерное распределение $\mu^{n}$ на $X_{n}$. Пусть

$$
\begin{gathered}
f \in C^{1}([0,1]), \quad \int_{0}^{1} f(x) d x=\rho<\frac{1}{2}, \\
f(0)=1, f(1)=0, f^{\prime}(x) \leqslant 0, x \in[0,1] .
\end{gathered}
$$

Следующая теорема формулирует вариационный принщип для этой задачи; лагранжиан напоминает энтропию.

Теорема 6.1. Имеет место равенство

əде

$$
\lim _{\varepsilon \rightarrow 0} \lim _{n} \frac{1}{\sqrt{n}} \ln \mu_{n}\left\{\varphi \in X_{n}:|\varphi-f|<\varepsilon\right\}=\int_{0}^{1} G\left(\sqrt{2\left|f^{\prime}(t)\right|}\right) d t,
$$

$$
G(u)=(2 / u+u) \ln \left(1-e^{-c(u+2 / u)}\right)-u \ln \left(1-e^{-c u}\right)-(2 / u) \ln \left(1-e^{-2 c / u}\right),
$$

а с - критическал точка функции $G$.

Аналогичный вариационный принцип можно сформулировать для всей полуоси. В данной задаче (о предельной конфигурации для разбиений с диаграммами, лежащими в квадрате) вариационный принцип справедлив, т. е. предельная конфигурация действительно есть единственное решение вариационной задачи.

ТЕорема 6.2. Предельная форма из теоремь 6.1 есть единственное решение вариачионной задачи на максимум с лагранжианом (6.1).

Интересно сравнить этот лагранжиан с тем, который возникает, если потребовать дополнительно выпуклость функций $f, f^{\prime \prime}(x) \geqslant 0$, и рассматривать лишь 
такие диаграммы, линейная верхняя огибающая которых также выпукла. В этом случае вариационная задача сформулирована в [8] и лагранжиан выглядит так:

$$
\int_{0}^{1} f^{\prime \prime}(t)^{1 / 3} d t \equiv \int_{\Gamma_{f}} \varkappa^{1 / 3} d s
$$

где $\varkappa$ - кривизна графика $\Gamma_{f}$ функции $f . \mathrm{B}[8]$ доказано, что и там вариационный принцип справедлив.

\section{ЛИТЕРАТУРА}

1. Vershik A. M. Asymptotic Combinatorics and Algebraic Analysis. Proceedings of ICM, Vol. 2, Birkhäuser, Zurich, 1995.

2. Arratia R., Tavare S. Limit theorems for combinatorial structutes. Rand. Structure Alg., 3, 321-345 (1992).

3. Fristedt $B$. The structure of random partitions of large integers. Trans. Am. Math. Soc., 337, 703-735 (1993).

4. Piman J., Aldous R. Brownian bridge asymptotics for random mappings. Rand. Structure Alg., 5, 487-512 (1994).

5. Хуанг К. Статистическая механика. Мир, М., 1966.

6. Хинчин А. Я. Математические основания квантовой статистики. Гостехиздат, M.-Л., 1951.

7. Freiman $G$. New analytical results in subset-sum problem. Combinatorics and Algorithms, Jerusalem, 1988.

8. Вершик A. M. Предельная форма выпуклых целочисленных многоугольников и близкие вопросы. Функц. анализ и его прил., 28, вып. 1, 17-25 (1994).

9. Barany $I$. The limits shape of convex lattice polygones. Discrete Comput. Geom., 13, 279-295 (1995).

10. Синай Я. Г. Вероятностный подход к анализу статистики выпуклых ломаных. Функц. анализ и его прил., 28, вып. 2, 41-48 (1994).

11. Vershik A. M. Statistics of the set of natural's, partitions. In: Probab. Theory Math. Stat., Vol. 2, 683-694, VNU Sci. Press.

12. Якубович Ю. Асимптотика случайных разбиений множеств. Зап. научн. семин. ЛОМИ, 223, 227-250 (1995).

13. Эндрюс Дж. Теория разбиений. Мир, М., 1980.

14. Siegel C. L. Lecture on advanced analytic number theory. Tata Inst. Bombay, 1961.

15. Szalay M., Turan $R$. On some problems of statistical theory of partitions. I. Acta Math. Acad. Sci. Hungr., 29, 361-379 (1977).

16. Вериик A. M., Керов C. В. Асимптотика максимальной и типичной размерности неприводимых представлений симметрической группы. Функц. анализ и его прил., 19, вып. 1, 25-36 (1985).

17. Рыжик И. М., Градитейн И. С. Таблицы интегралов, сумм, рядов, произведений. Физматгиз, М., 1963.

18. Вериик A. М., Шмидт A. А. Предельные меры, возникающие в асимптотической теории симметрических групп. Теор. вер. и прим., 22, вып. 1, 72-88 (1977); 23, вып. 1, 42-54 (1978).

19. Вершик A. M. Статистическая сумма, связанная с диаграммами Юнга. Зап. научн. сем. ЛОМИ, 164, 20-29 (1987). 